\title{
Evidence of Trigonal Dangling Bonds at the Ge(111)/Oxide Interface by Electrically Detected Magnetic Resonance
}

\author{
S. Paleari \\ Dipartimento di Scienza dei Materiali, Università degli Studi di Milano Bicocca, via Cozzi 53, I-20125 Milan, Italy \\ S. Baldovino and A. Molle \\ Laboratorio MDM, IMM-CNR, Via C. Olivetti 2, I-20864 Agrate Brianza (MB), Italy \\ M. Fanciulli* \\ Dipartimento di Scienza dei Materiali, Università degli Studi di Milano Bicocca, via Cozzi 53, I-20125 Milan, \\ Italy and Laboratorio MDM, IMM-CNR, Via C. Olivetti 2, I-20864 Agrate Brianza (MB), Italy \\ (Received 21 January 2013; revised manuscript received 28 March 2013; published 15 May 2013)
}

\begin{abstract}
Despite a renewed interest in $\mathrm{Ge}$ as a competitor with $\mathrm{Si}$ for a broad range of electronic applications, the microstructure and the electronic properties of the dangling bonds that, in analogy with $\mathrm{Si}$, are expected at the Ge/oxide interface have escaped a firm spectroscopy observation and characterization. Clear evidence based on contactless electrically detected magnetic resonance spectroscopy of a dangling bond at the $\mathrm{Ge}(111) / \mathrm{GeO}_{2}$ interface is reported in this Letter. This result supports the similarity between dangling bonds at the $\mathrm{Si}(111)$ /oxide and $\mathrm{Ge}(111)$ /oxide interfaces, both showing $\mathrm{C}_{3 v}$ trigonal point symmetry with the main axis oriented along the $\langle 111\rangle$ direction. In contrast, at the $\mathrm{Ge}(001) /$ oxide interface the absence of the trigonal center in favor of a lower symmetry dangling bond marks the difference with the $\mathrm{Si}(001) /$ oxide interface, where both centers are present and the one having higher point symmetry prevails. This fact is rationalized in terms of suboxide interface rearrangement and oxide viscoelasticity, which promote the generation of the nonaxial centers at distorted dimers. The unambiguous identification of the centers at the Ge/oxide interfaces yields a deeper insight into the physical properties of the suboxide interface structure and offers a valid indicator for the evaluation of different surface capping and passivation techniques, with the potential to boost the Ge-related technology.
\end{abstract}

DOI: 10.1103/PhysRevLett.110.206101

PACS numbers: 68.35.bg, 73.20.Hb, 76.30.-v

In the quest for improving the performance of electronic devices, recently germanium emerged as a candidate for postsilicon electronic applications. These include nanoscaled digital devices, spintronics, photonics, photovoltaics, and optoelectronics [1-5] and rely on higher hole mobility, long spin coherence time, and accessibility to the direct gap. The control of electrically active defects on the Ge surface and at the interface with oxides is critical to exploit Ge as an active channel material for faster devices [6] and for optoelectronic functionalities [7]. In analogy with $\mathrm{Si}$, an important role in determining Ge surface properties is played by the dangling bond (DB). Both theoretical and experimental arguments [8-10] indicate that the Ge DB is a charge trapping center responsible for the density of states at the interface between $\mathrm{Ge}$ and oxides and possibly for the Fermi level pinning. Despite its impact, little is known on the microstructure of the $\mathrm{DB}$ at the $\mathrm{Ge} /$ oxide interface. In this context, solid evidence of the $\mathrm{Ge}$ $\mathrm{DB}$ at the $\mathrm{Ge}(001) / \mathrm{Ge}$ oxide interface has been recently reported [11,12]. Electrically detected magnetic resonance (EDMR) spectroscopy revealed a nontrigonal symmetry of the Ge DB at the (001) oriented interface with its elemental oxide, therein resembling a similar finding at the (001) $\mathrm{Si}_{x} \mathrm{Ge}_{1-x} / \mathrm{SiO}_{2}$ interface [13,14], which, however, shows a different point symmetry. The lack of experimental evidence reporting a trigonal DB at the $\mathrm{Ge}(001) / \mathrm{GeO}_{2}$ interface is somehow surprising. Indeed, under thermal oxidation the $\mathrm{Ge}(001)$ surface is supposed to reorganize similarly to its $\mathrm{Si}(001)$ counterpart, where two different paramagnetic centers are simultaneously present $[15,16]$. The more abundant DB, the $P_{b 0}$ drawn in Fig. 1(b), is a trigonal center, whereas the other reported $\mathrm{DB}$, the $P_{b 1}$ drawn in Fig. 1(c), is monoclinic. In the case of the $\operatorname{Si}(111)$ surface, only the trigonal DB, named $P_{b}$, is reported [see Fig. 1(a)]. In this Letter, new light is shed on this matter by the unambiguous observation of an electrically active paramagnetic center at the $\mathrm{Ge}(111) / \mathrm{GeO}_{2}$ interface with an axial $g$ matrix and a principal axis along the [111] direction. Hence, a simple analysis of the role of the suboxide asymmetric dimer in the Ge DB microstructure is envisioned, starting from a comparative EDMR analysis of the thermally oxidized $\mathrm{Ge}(001)$ and $\mathrm{Ge}(111)$ surfaces.

Highly resistive (nominal resistivity $48 \Omega \mathrm{cm}$ ) $\mathrm{Ge}(001)$ and $\mathrm{Ge}(111)$ monocrystal substrates were chemically cleaned with HF dipping and then inserted in a rapid thermal annealing apparatus for a 20-min-long vacuum heating at $400{ }^{\circ} \mathrm{C}$ in an ultrapure $\mathrm{O}_{2}$ overpressure. 


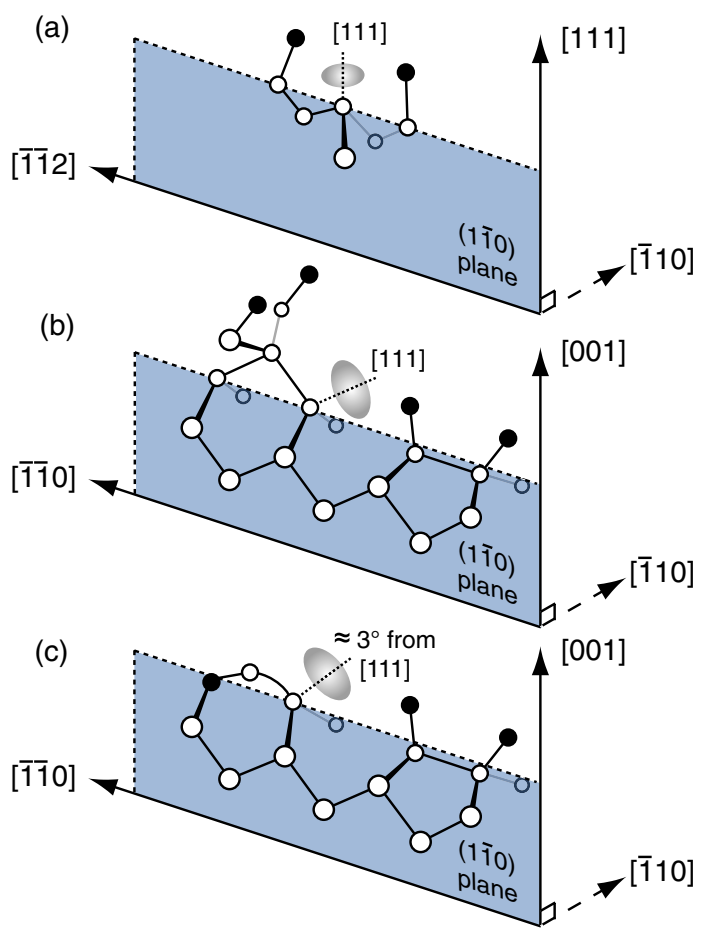

FIG. 1 (color online). Schematic picture of the currently accepted dangling bond configuration at $\mathrm{Si} / \mathrm{SiO}_{2}$ surfaces $[15,16]$ : (a) trigonal $P_{b}$ center at the (111) surface and (b), (c) trigonal $P_{b 0}$, and nontrigonal $P_{b 1}$, respectively, both at the (001) surface. The $P_{b 1}$ is drawn according to the asymmetrically oxidized dimer model of Ref. [15]. Open circles represent Si atoms, filled circles represent $\mathrm{O}$ atoms, and the DB is sketched by the shaded ellipsoid.

Compositional aspects on the Ge oxidation were assessed by means of x-ray photoelectron spectroscopy indicating a dominant $\mathrm{GeO}_{2}$ contribution in analogy to what is observed in the case of the $\mathrm{Ge}(001)$ thermal oxidation [17]. EDMR spectroscopy was performed in contactless mode and was specifically preferred over conventional spin resonance, because a significantly higher sensitivity to interface traps can be achieved by probing spin-dependent variation in the photoconductivity due to electron-hole pair recombination assisted by defect states [18-21]. A lock-in technique was employed, by introducing a magnetic field modulation. In detail, the sample was placed at the center of a Bruker electric field cavity (ER4109EF) operating in the $X$ band and illuminated with a blue light-emitting diode $(\lambda \approx 450 \mathrm{~nm})$ through an optical window. In this position, the sample experiences the microwave electric field component, and, as a consequence, spin-dependent changes of the photocurrent induce a detectable change in the cavity $Q$ factor, allowing the identification of the electrically active defects. The measurements were taken at the temperature of $5 \mathrm{~K}$, with a microwave frequency of about $9.2 \mathrm{GHz}$ and a $7 \mathrm{kHz}$ magnetic field modulation of sufficiently low amplitude to avoid signal distortion. Additional experimental details can be found elsewhere [21].
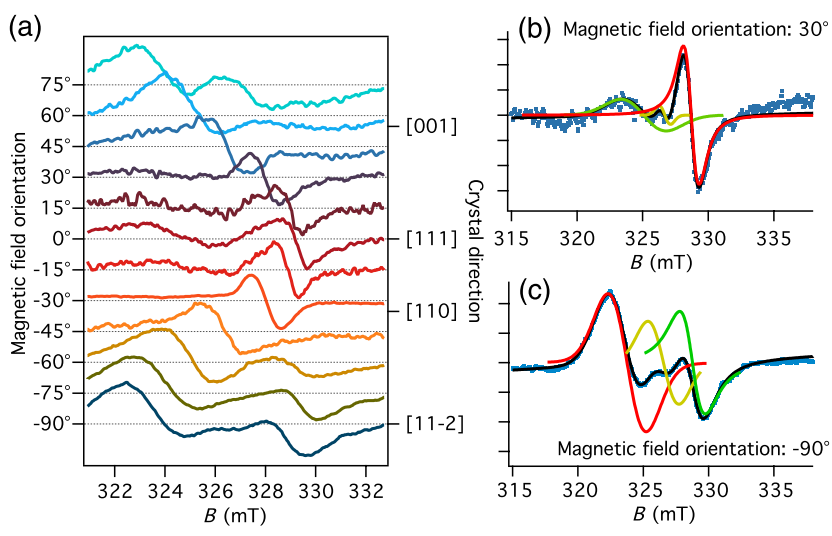

FIG. 2 (color online). (a) Angular dependence of the first derivative-EDMR spectra of the $(111) \mathrm{Ge} / \mathrm{GeO}_{2}$ sample. The vector $\mathbf{B}$ lies in the (110) plane, and the angle is referred to the surface normal [cf. Fig. 1(a)]. After a second-order polynomial background subtraction, the various spectra have been rescaled in the abscissa to account for the small differences in microwave frequency and normalized in intensity, for a better visual comparison. (b), (c) Best fit of representative spectra, with an angle between the magnetic field and the surface normal of $30^{\circ}$ and $90^{\circ}$, respectively. The experimental points are the blue dots, and the fitted spectra are the black lines. The deconvolution of the different branches is evidenced. In (b), the [111] branch (red line) is the last and the other $\langle 111\rangle$ branches are the first and the second, from left to right (green and yellow lines, respectively). In (c), the [111] is the first and the other $\langle 111\rangle$ are the second and the third. For the references to the colors, the reader is referred to the Web version of this Letter.

Figure 2(a) shows a collection of the spectra recorded at different magnetic field orientations with respect to the sample crystal axis. Best fits of representative spectra are shown in Figs. 2(b) and 2(c). As the B field was rotated in the (110) plane, a distinct pattern was observed. Each spectrum was fitted with the minimal number of Voigt lines, from which the resonant field and the linewidth of the single components were extracted. The $g$ factor is calculated from the resonance condition $h \nu=g \mu_{B} \mathbf{B}_{0}$, where $\nu$ is the microwave frequency, $\mu_{B}$ is the Bohr magneton, and $\mathbf{B}_{0}$ is the line center position [22]. The $g$ factor reflects the local environment of the paramagnetic center and carries important information about the symmetry.

Figure 3 shows the $g$-matrix road map, as extracted from the deconvolution of the EDMR spectra of Fig. 2. A welldefined pattern is present, allowing the identification of the main directions and principal $g$ values. Considered the degeneracy expected for an axial center oriented along the $\langle 111\rangle$ direction within the Ge lattice [cf. Fig. 1(a)], the most intense branch of the $g$ pattern is associated with those centers having the symmetry axis parallel to the $\mathrm{Ge}$ surface normal [111], depicted in red in Fig. 3. This finding is in line with EPR data of defects at the $\mathrm{Si}(111) / \mathrm{SiO}_{2}$ interface [23], and it is consistent with defects located at the Ge(111)/oxide interface. Each branch can be fitted by 
Crystal direction

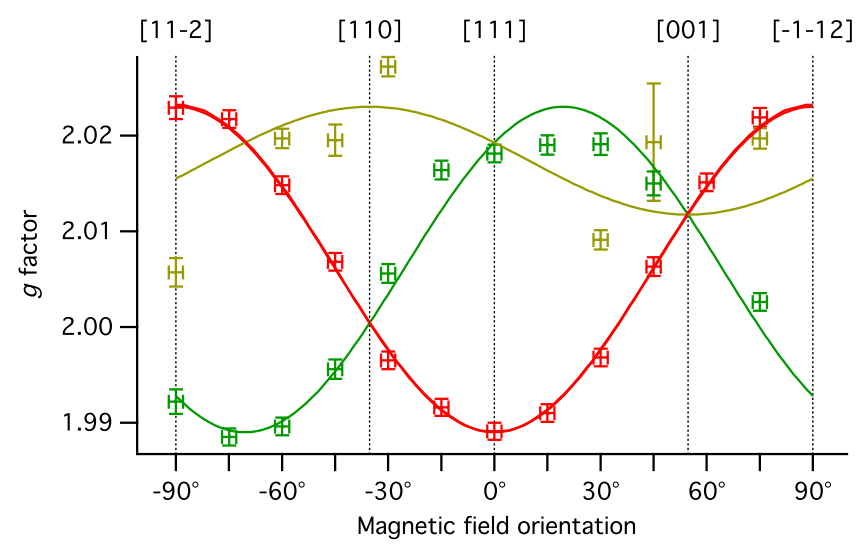

FIG. 3 (color online). Angular $g$ map of the $\mathrm{GeO}_{2} / \mathrm{Ge}(111)$ DB observed by the contactless EDMR technique at $9.2 \mathrm{GHz}$ and $5 \mathrm{~K}$ for $\mathbf{B}$ rotating in the (1 10$)$ plane. The solid curves represent the best fitting of each branch to a trigonal symmetry in center in a Ge (diamond) crystal, yielding the principal $g$ values $g_{\|}=$ $1.9890 \pm 0.0004$ and $g_{\perp}=2.0230 \pm 0.0004$. The branches are color coded as in Fig. 2.

$g=\sqrt{g_{\|}^{2} \cos ^{2} \theta+g_{\perp}^{2} \sin ^{2} \theta}$, where $\theta$ is the angle between $\mathbf{B}$ and the paramagnetic center axis [22]. The experimental data reported in Fig. 3 are consistent with a $\mathrm{C}_{3 v}$ point symmetry, and $g_{\|}=1.9890 \pm 0.0004, g_{\perp}=2.0230 \pm$ 0.0004 with the principal axis oriented along the $\langle 111\rangle$ direction.

The $g$-factor principal values strongly support the assignment to an interfacial Ge DB. Starting from a comparison with the well-known $P_{b}$ center at the $\mathrm{Si}(111) / \mathrm{SiO}_{2}$ interface, where simple first-order spin-orbit theory predicts $g_{\perp}-g_{\text {f.e. }} \propto \lambda\left(g_{\text {f.e. }}=2.00232 \ldots\right.$ is the free electron $g$ value and $\lambda$ is the spin-orbit coupling constant), it can be noticed that $\left[g_{\perp}-g_{\text {f.e. }}\right](\mathrm{Ge} \mathrm{DB}) /\left[g_{\perp}-g_{\text {f.e. }}\right] \times$ $\left(\mathrm{Si} P_{b 0}\right)$ is well in line with the ratio $\lambda_{\mathrm{Ge}} / \lambda_{\mathrm{Si}}=$ $940 \mathrm{~cm}^{-1} / 142 \mathrm{~cm}^{-1}$ [13,23-25]. Another solid argument in favor of the assignment comes from the results obtained on $\mathrm{Ge}(001)$ with the contactless EDMR technique, showing DB activity at the Ge/oxide interface [11]. The observed $g$ values were in the range 1.9922-2.0238, which is in a fairly good agreement with the present findings (cf. Table I). The large linewidths (1-3 mT) also concur with the previous Ge DB data $[11,24,26]$.

TABLE I. Comparison between the $g$ factors observed at $\mathrm{Ge}(111) / \mathrm{GeO}_{2}$ [this work] and $\mathrm{Ge}(100) / \mathrm{GeO}_{2}$ [11] interfaces.

\begin{tabular}{lcc}
\hline \hline Interface type & $g_{\|}$ & $g_{\perp}$ \\
\hline $\mathrm{Ge}(111) / \mathrm{GeO}_{2}$ & $1.9890(4)$ & $2.0230(4)$ \\
\hline $\mathrm{Ge}(001) / \mathrm{GeO}_{2}{ }^{\mathrm{a}}$ & $1.9922(3)$ & $2.0238(3)$ \\
\hline \hline
\end{tabular}

${ }^{a}$ As no clear symmetry axis could be inferred in this case, the extremal $g$ values are reported for comparison.
The interfacial nature of the observed DB is further evidenced by the complete suppression of the signal upon etching of the $\mathrm{GeO}_{2}$ in deionized water. Following the etching procedure, for short exposure to air, when the native oxide had not enough time to grow, the signal is absent while it is recovered for longer exposures to air [27]. As the strong anisotropy rules out the location inside the amorphous oxide matrix, the center must be placed at the interface.

An additional observation in support of the interfacial DB assignment is the inhomogeneous line broadening shown in Fig. 4, resulting from a strain-induced spread $\sigma g(\theta)$, observed mainly in $g_{\perp}$, as for the case of the $\mathrm{Si} /$ oxide interface. The standard deviation of $\sigma g$ can be characterized by extracting the width $\Delta B_{\mathrm{pp}}^{G}(\theta)$ of the Gaussian line broadening from the total width $\Delta B_{\mathrm{pp}}^{T}(\theta)$ of a Voigt function [25]:

$\Delta B_{\mathrm{pp}}^{T}(\theta)=1 / 2 \Delta B_{\mathrm{pp}}^{L}+\sqrt{\left[\Delta B_{\mathrm{pp}}^{G}(\theta)\right]^{2}+1 / 4\left(\Delta B_{\mathrm{pp}}^{L}\right)^{2}}$,

where $\Delta B_{\mathrm{pp}}^{T}(\theta=0)$ is taken as the homogeneous Lorentzian width $\Delta B_{\mathrm{pp}}^{L}$, assumed to be independent of $\theta$. $\sigma g$ can then be obtained by fitting $\Delta B_{\mathrm{pp}}^{G}(\theta)$ using the formula

$\Delta B_{\mathrm{pp}}^{G}(\theta)=\left(2 h \nu / g^{3} \mu_{B}\right)\left(g_{\|} \cos ^{2} \theta \sigma_{g_{\|}}+g_{\perp} \sin ^{2} \theta \sigma_{g_{\perp}}\right)$.

A fit to the data yields $\sigma_{g_{\perp}}=0.0066 \pm 0.0002$, again approximately $\lambda_{\mathrm{Ge}} / \lambda_{\mathrm{Si}}$ times larger than the value observed for $\mathrm{Si} P_{b}\left(\sigma_{g_{\perp}} \approx 0.00085\right)$ [25]. This result is also in good agreement with previous measurements on the Ge $\mathrm{DB}$ at the $\mathrm{Si}_{x} \mathrm{Ge}_{1-x} / \mathrm{SiO}_{2}$ interface giving $\sigma_{g_{\perp}} \approx$ 0.0074 [13].

The role of the surface orientation invokes a nontrivial interpretation of the Ge DB nature which should be related to the details of the oxidation process and its interplay with the suboxide bond net. By restricting our analysis to the DBs, it can be noticed that $\mathrm{Si}$ and Ge exposing the (111)

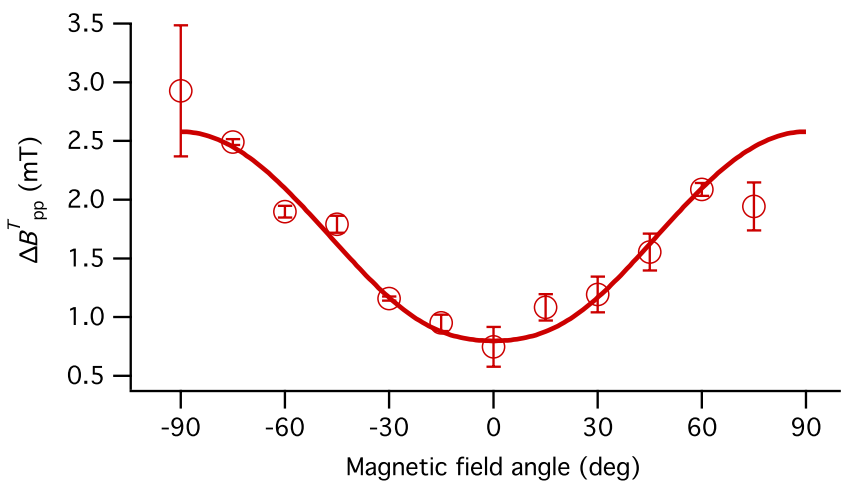

FIG. 4 (color online). EDMR peak-to-peak linewidth $\Delta B_{\mathrm{pp}}^{T}$ of the [111] DB as a function of the angle between $\mathbf{B}$ and the paramagnetic center principal axis. The solid line represents a fit based on Eq. (2), from which the value $\sigma_{g_{\perp}}=0.0066 \pm 0.0002$ is deduced. 
surface are quite similar, with a prominent axially symmetrical center due to the structural mismatch between the crystal lattice and the amorphous oxide [cf. Fig. 1(a)]. On the other hand, by looking at the (001) surface, the differences between oxidized $\mathrm{Si}$ and $\mathrm{Ge}$ can be rationalized in terms of trigonal, $P_{b 0}$-like, and nontrigonal, $P_{b 1}$-like, centers, with the first oriented in the $\langle 111\rangle$ direction and the second tilted off [cf. Figs. 1(b) and 1(c), respectively]: While they are both present in $\mathrm{Si}$ (with a prevalence of the $P_{b 0}$ ), only nontrigonal DBs can be observed in Ge. No $P_{b 0}$-like has been reported in the case of Ge/oxide interfaces by angular resolved EPR or EDMR; nevertheless, evidence of a trigonal Ge DB with the main axis along the [111] direction has been found in other systems such as weakly oxidized epitaxial porous $\mathrm{Si}_{0.8} \mathrm{Ge}_{0.2}$ [24] or O-implanted Si-Ge alloys [28]. In the latter case, the $g$ values of the center depend on the Ge contents of the alloy.

It is quite well established, at least in the case of $\mathrm{Si}$, that the lower symmetry of the nontrigonal center is related to the distortion introduced in the DB wave function by strained bonds in the close neighborhood [15]. In turn, the strained bond is formed upon asymmetrical oxidation of dimer structures existing in the suboxide region. Actually, both $\mathrm{Si}$ and Ge unoxidized (001) surfaces reconstruct in dimers with a dominance of $(2 \times 1)$ periodicity. $\mathrm{Ge}$ is more thermodynamically prone to form asymmetric dimers- $c(4 \times 2)$ or $p(2 \times 2)$ reconstructions-due to bond buckling even at room temperature [29,30], while $\mathrm{Si}$ exhibits stable buckled structures only in correspondence with steps and defects or at low temperatures [31,32]. At least in the case of Si, this tendency is retained even at the oxidized subinterface, where a transition layer is responsible for dimer reconstruction at the buried interface [33]. A similar mechanism in Ge could be responsible for the nontrigonal structure observed at the (001) surface: The partial subinterface reconstruction may promote the backbond oxidation at dimer sites. The absence of the trigonal counterpart could be tentatively explained in terms of the surface relaxation argument. Recent results [16] on $\mathrm{Si}(001) / \mathrm{SiO}_{2}$ assign the $P_{b 0}$ to a DB located at a monolayer step with a dimerized upper part [cf. Fig. 1(b)—such steps are always likely to occur in samples due to slight offaxis cut and surface roughness]. This DB structure could be mainly suppressed in $\mathrm{Ge}$, because of the higher viscosity of the $\mathrm{GeO}_{2}$ [34] which allows stress relaxation without bond breaking. At the Ge/oxide interface very low density of interface traps is observed, indeed, in comparison to the unpassivated $\mathrm{Si} /$ oxide interface.

A complementary explanation for the lack of EDMR detection of the trigonal DB at the Ge(001) suboxide interface could be related to the position of the charging levels of this center, since it is paramagnetic only in the neutral state [9]. Differences in the charging level scheme, associated with the surface orientation, could reduce the $P_{b 0}$-like signal, below the electrical detection threshold
[35]. However, at the moment, this hypothesis is unsupported, and no orientation dependence of the electronic levels of the suboxide traps is reported for Ge by standard electrical characterization [10,36].

In conclusion, by exploiting the enhanced sensitivity allowed by the electrical detection of electron paramagnetic resonance, a trigonal dangling bond has been unequivocally identified at the $\mathrm{Ge}(111) /$ oxide interface, with the main axis oriented along the $\langle 111\rangle$ direction. This result reveals a close similarity between the (111)oriented $\mathrm{Si}$ and $\mathrm{Ge}$ oxidized interfaces, both showing the same DB microstructure. On the other hand, in the case of the (001)-oriented surfaces $\mathrm{Si}$ shows the trigonal $\langle 111\rangle$-oriented $P_{b 0}$ and the nontrigonal $P_{b 1}$, whereas only a nontrigonal center manifests at the $\mathrm{Ge}(001) /$ oxide interface. In this case, the presence of a distorted dimer net in the suboxide region could favor the lower symmetry DB, while the occurrence of the axial counterpart could be hampered by the higher viscosity of the oxide. The unambiguous identification of the center can be used to characterize different surface capping and passivation techniques, which may overcome drawbacks of the Ge-based technology and push it beyond Si performances.

*marco.fanciulli@unimib.it

[1] R. Pillarisetty, Nature (London) 479, 324 (2011).

[2] J. Xiang, W. Lu, Y. Hu, Y. Wu, H. Yan, and C. M. Lieber, Nature (London) 441, 489 (2006).

[3] A. Jain et al., Phys. Rev. Lett. 109, 106603 (2012).

[4] S. Assefa, F. Xia, and Y. A. Vlasov, Nature (London) 464, 80 (2010).

[5] J. Kouvetakis and A. V. G. Chizmeshya, J. Mater. Chem. 17, 1649 (2007).

[6] Y. Kamata, Mater. Today 11, 30 (2008).

[7] V.R. D'Costa, Y.-Y. Fang, J. Tolle, J. Kouvetakis, and J. Menéndez, Phys. Rev. Lett. 102, 107403 (2009).

[8] A. Dimoulas, P. Tsipas, A. Sotiropoulos, and E. K. Evangelou, Appl. Phys. Lett. 89, 252110 (2006).

[9] P. Broqvist, A. Alkauskas, and A. Pasquarello, Phys. Rev. B 78, 075203 (2008).

[10] D. Kuzum, A. J. Pethe, T. Krishnamohan, and K. C. Saraswat, IEEE Trans. Electron Devices 56, 648 (2009).

[11] S. Baldovino, A. Molle, and M. Fanciulli, Appl. Phys. Lett. 93, 242105 (2008).

[12] S. Baldovino, A. Molle, and M. Fanciulli, Appl. Phys. Lett. 96, 222110 (2010).

[13] A. Stesmans, P. Somers, and V. V. Afanas'ev, Phys. Rev. B 79, 195301 (2009).

[14] P. Somers, A. Stesmans, L. Souriau, and V. V. Afanas'ev, J. Appl. Phys. 112, 074501 (2012).

[15] A. Stirling, A. Pasquarello, J. Charlier, and R. Car, Phys. Rev. Lett. 85, 2773 (2000).

[16] F. Hoehne, J. Lu, A. R. Stegner, M. Stutzmann, M. S. Brandt, M. Rohrmüller, W. G. Schmidt, and U. Gerstmann, Phys. Rev. Lett. 106, 196101 (2011). 
[17] See Supplemental Material at http://link.aps.org/ supplemental/10.1103/PhysRevLett.110.206101 and references therein for additional details.

[18] D. J. Lepine, Phys. Rev. B 6, 436 (1972).

[19] E.H. Pointdexter, P.J. Caplan, B.E. Deal, and R.R. Razouk, J. Appl. Phys. 52, 879 (1981).

[20] L.S. Vlasenko, Y. V. Martynov, T. Gregorkiewicz, and C. A. J. Ammerlaan, Phys. Rev. B 52, 1144 (1995).

[21] M. Fanciulli, O. Costa, S. Cocco, G. Seguini, E. Prati, and G. Scarel, in NATO Advanced Studies Institute, Series II: Mathematics, Physics and Chemistry (Plenum, New York, 2005), p. 26.

[22] J.A. Weil and J.R. Bolton, Electron Paramagnetic Resonance (Wiley, New York, 1994).

[23] A. Stesmans, Appl. Phys. Lett. 48, 972 (1986).

[24] S. Lebib, M. Schoisswohl, J.L. Cantin, and H. J. von Bardeleben, Thin Solid Films 294, 242 (1997).

[25] A. Stesmans and V. Afanas'ev, J. Appl. Phys. 83, 2449 (1998).

[26] T. Graf, T. Ishikawa, K. M. Itoh, E. E. Haller, M. Stutzmann, and M. S. Brandt, Phys. Rev. B 68, 205208 (2003).
[27] See Supplemental Material at http://link.aps.org/ supplemental/10.1103/PhysRevLett.110.206101 for the experimental spectra.

[28] M. Zvanut and W. Carlos, J. Vac. Sci. Technol. B 10, 2026 (1992).

[29] O. Gurlu, H. J. W. Zandvliet, and B. Poelsema, Phys. Rev. Lett. 93, 066101 (2004).

[30] H. J. Zandvliet, Phys. Rep. 388, 1 (2003).

[31] H. Zandvliet, Rev. Mod. Phys. 72, 593 (2000).

[32] P. Bokes, I. Štich, and L. Mitas, Chem. Phys. Lett. 362, 559 (2002).

[33] A. Pasquarello, M. Hybertsen, and R. Car, Nature (London) 396, 58 (1998).

[34] M. Houssa, G. Pourtois, M. Caymax, M. Meuris, M. M. Heyns, V. V. Afanas'ev, and A. Stesmans, Appl. Phys. Lett. 93, 161909 (2008).

[35] J. P. Campbell and P. M. Lenahan, Appl. Phys. Lett. 80, 1945 (2002).

[36] T. Sasada, Y. Nakakita, M. Takenaka, and S. Takagi, J. Appl. Phys. 106, 073716 (2009). 